\title{
Effects of bispectral index monitoring as an adjunct to nurse-administered propofol combined sedation during colonoscopy: a randomized clinical trial
}

Jun $\mathrm{Heo}^{1}$, Min Kyu Jung ${ }^{1}$, Hyun Seok Lee ${ }^{1}$, Chang Min Cho ${ }^{1}$, Seong Woo Jeon ${ }^{1}$, Sung Kook Kim , and Young Hoon Jeon ${ }^{2}$

Departments of ${ }^{1}$ Internal Medicine and ${ }^{2}$ Anesthesiology and Pain Medicine, Kyungpook National University School of Medicine, Daegu, Korea

Received: April 5, 2014

Revised : February 11, 2015

Accepted: April 5, 2015

\section{Correspondence to}

Min Kyu Jung, M.D.

Department of Internal Medicine, Kyungpook National University

School of Medicine, 680

Gukchaebosang-ro, Jung-gu,

Daegu 41944, Korea

Tel: +82-53-420-5514

Fax: +82-53-426-8773

E-mail: minky1973@knu.ac.kr
Background/Aims: The efficacy of bispectral index (BIS) monitoring during colonoscopic sedation is debated. We aimed to determine whether BIS monitoring was useful for propofol dose titration, and to evaluate differences in sedative administration between expert and inexperienced medical personnel during colonoscopy procedures that required moderate sedation.

Methods: Between February 2012 and August 2013, 280 consecutive patients scheduled to undergo a screening colonoscopy participated in this study and were randomly allocated to the expert or inexperienced endoscopist group. Each group was further divided into either a BIS or a modified Observer's Assessment of Alertness/Sedation Scale (MOAA/S) subgroup. Trained nurses administered combined propofol sedation and monitored sedation using either the BIS or MOAA/S scale.

Results: The mean BIS value throughout the procedure was $74.3 \pm 6.7$ for all 141 patients in the BIS group. The mean total propofol dose administered in the BIS group was higher than that in the MOAA/S group, independently of the endoscopists' experience level $(36.9 \pm 29.6$ and $11.3 \pm 20.7$, respectively; $p<0.001)$. The total dose of propofol administered was not significantly different between the inexperienced endoscopist group and the expert endoscopist group, both with and without the use of BIS ( $p=0.430$ and $p=0.640$, respectively).

Conclusions: Compared with monitoring using the MOAA/S score alone, BIS monitoring was not effective for titrating the dose of propofol during colonoscopy, irrespective of colonoscopist experience.

Keywords: Conscious sedation; Consciousness monitors; Sedative colonoscopy

\section{INTRODUCTION}

A colonoscopy is uncomfortable for the patient because of its long duration and the use of a larger-diameter en- doscope that is routinely used for upper gastrointestinal endoscopy [1]. In addition, loop formation in the colon occurs frequently during the procedure. Therefore, sedation and analgesia are typically required to improve 
patient tolerance and compliance during the colonoscopy procedure [2]. However, achieving and maintaining an adequate level of sedation is difficult. Patients frequently become agitated when low levels of sedative drugs are used; however, adverse events such as respiratory distress and lack of patient cooperation can occur when an excessive dose of sedative is administered. Therefore, it is important to set an objective indicator for monitoring the patient sedation level during colonoscopy, especially when performed by inexperienced endoscopists.

Electroencephalography (EEG)-guided sedation has been used by anesthesiologists to achieve optimal titration of sedatives [3]. Bispectral index (BIS) monitoring is an EEG-based method that quantifies the depth of anesthesia by analyzing the EEG and uses a complex algorithm to generate an index score, which provides an objective measurement of the level of consciousness in sedated patients [3]. Previously, we validated the efficacy and safety of nurse-administered combined sedation using BIS monitoring during endoscopic retrograde cholangiopancreatography (ERCP) [4]. However, the value of BIS monitoring as an adjunct to colonoscopic sedation has been tested in only a limited number of colonoscopic studies [5-10].

The objectives of the current study were to determine whether BIS monitoring was useful for the titration of propofol in combined sedation, and to compare the efficacy of BIS monitoring performed by expert and inexperienced endoscopists during colonoscopy.

\section{METHODS}

\section{Study design and participants}

This prospective, randomized, controlled, open-label trial was conducted in Kyungpook National University Hospital between February 2012 and August 2013. The Institutional Review Board of Kyungpook National University Hospital Ethics Committee approved this study. The trial was conducted in accordance with good clinical practice under the principles of the Declaration of Helsinki.

Consecutive patients who required a colonoscopy screening participated in the study. Informed consent was obtained from all of the participating patients. Ex- clusion criteria included age $<18$ years, critical illness (defined by the American Society of Anesthesiologist [ASA] as class 4 or 5) [11], pregnancy, long-term use of benzodiazepines or opiates, history of allergy to eggs, past history of abdominal surgery, and other contraindications for endoscopy such as uncooperativeness, or signs of peritonitis.

\section{Randomization and intervention}

The randomization of patients was performed using the SPSS version 21.o (IBM Co., Armonk, NY, USA). The patients were randomly allocated into either the expert or inexperienced endoscopist group. Endoscopists were considered expert if they had performed more than 300 colonoscopies, while inexperienced endoscopists had completed fewer than 100 colonoscopies [12]. A total of 10 endoscopists (five experienced and five inexperienced) participated in the study. Each endoscopist experience level group was further divided into either a BIS or a modified Observer's Assessment of Alertness/ Sedation Scale (MOAA/S) subgroup. The endoscopist, trained nurse, and assistant nurse had access to the randomization scheme when the patient was admitted to the endoscopy suite. Three trained nurses administered the sedatives and performed the anesthetic protocol formulated by an experienced anesthesiologist (YHJ, who has performed $>1,000$ general anesthesia procedures per year for the past 20 years). The patients received 0.03 to $0.06 \mathrm{mg} / \mathrm{kg}$ midazolam and $50 \mathrm{mg}$ pethidine prior to the procedure. A trained nurse assessed the sedation grade using BIS monitoring or the MOAA/S scale every 3 minutes [6] and intermittently administered 10 to 20 $\mathrm{mg}$ propofol to maintain conscious sedation [13]. For the MOAA/S group, the sedation end point was an MOAA/S score of three. For the BIS group, the nurse was instructed to target a BIS value between 70 and 80 as the primary end point for propofol titration.

\section{Monitoring}

All patients were continuously monitored for heart rate, oxygen saturation (pulse oximetry), and blood pressure (automated blood pressure cuff with serial measurements every 3 minutes). Baseline vital signs were recorded immediately before the procedure. All patients were given supplemental intranasal oxygen ( $2 \mathrm{~L} / \mathrm{min})$. Respiratory depression was considered significant when 
oxygen saturation was $<80 \%$ for $>15$ seconds with oxygen supplementation. A drop in systolic arterial blood pressure to below $80 \mathrm{mmHg}$, or a heart rate lower than 50 beats per minute, was considered to be a significant adverse event. All patients were monitored in the recovery area by electrocardiography, pulse oximetry, and blood-pressure recording. All patients in the BIS group were monitored for BIS scores using a BIS VIEW device (BIS monitoring system, Aspect Medical Systems, Norwood, MA, USA) and a specific BIS Quatro sensor (Aspect Medical Systems).

\section{Outcome measures}

Patients were moved to the recovery area immediately after the procedure if their vital signs were stable. An independent research fellow $(\mathrm{JH})$, who was blinded to the randomization scheme, performed all preprocedural and postprocedural assessments. Total dose of propofol and midazolam, procedure time, patient pain level during the colonoscopy, satisfaction level of patients and endoscopists, and adverse events were compared between the BIS group and the MOAA/S group. After the colonoscopy was completed, a questionnaire, which evaluated the degree of pain and satisfaction using a visual analog scale (VAS), was administered to the patients and endoscopists. The results of the patient questionnaire were confirmed through telephone consultation or visiting the outpatient unit 7 to 10 days following the colonoscopy procedure. A VAS was used to score pain (o, none; 10, severe) and satisfaction (o, poor; 10, excellent). Comparisons between the expert and inexperienced medical personnel groups were performed by per-protocol analyses.

\section{Sample size and statistical analysis}

To determine if a $0.02 \mathrm{mg} / \mathrm{min}$ per $\mathrm{kg}$ propofol dose difference (from 0.08 in the BIS group to 0.10 in the $\mathrm{MOAA} / \mathrm{S}$ group) was significant with a power of $0.8 \mathrm{o}$ and a two-sided $\alpha=0.05$, and standard deviation of $0.05 \mathrm{mg} /$ min per kg, we calculated that a minimum sample size of 51 patients was required for each experimental group. Assuming a dropout rate of $20 \%$, we determined that at least 248 patients were required to have four experimental groups, each with 62 patients. Continuous data were compared using a $t$ test and categorical variables were tested using the chi-square test. The criterion for statis- tical significance was $p<0.05$. The SPSS version 21.0 was used for the statistical analyses. Data are presented as mean \pm standard deviation.

\section{RESULTS}

\section{Baseline characteristics}

Excluding the 20 patients who could not tolerate cecal intubation during colonoscopy, 280 patients were analyzed. Among them, 149 were enrolled in the expert endoscopist group and 131 in the inexperienced endoscopist group (Fig. 1). No significant differences were observed among the subgroups in baseline characteristics including age, sex, body surface area, or ASA physical status (Table 1).

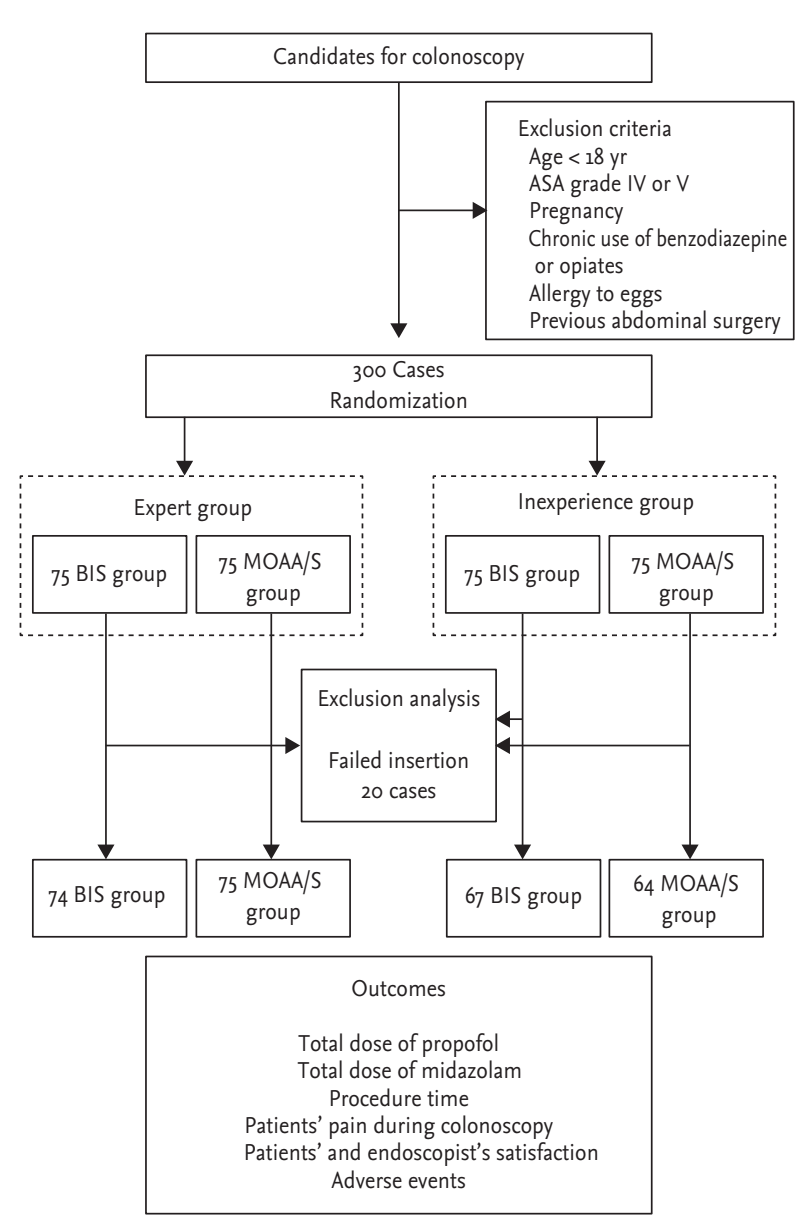

Figure 1. Flowchart of patient enrollment in the study. ASA, American Society of Anesthesiologists; BIS, bispectral index; MOAA/S, modified Observer's Assessment of Alertness/Sedation Scale. 
Table 1. Baseline characteristics of the patients

\begin{tabular}{|c|c|c|c|c|c|c|}
\hline \multirow{2}{*}{ Characteristic } & \multicolumn{3}{|c|}{ Expert endoscopist } & \multicolumn{3}{|c|}{ Inexpert endoscopist } \\
\hline & $\operatorname{BIS}(n=74)$ & MOAA/S $(\mathrm{n}=75)$ & $p$ value & $\operatorname{BIS}(n=67)$ & $\mathrm{MOAA} / \mathrm{S}(\mathrm{n}=64)$ & $p$ value \\
\hline Age, yr & $54 \cdot 3 \pm 13.8$ & $56.3 \pm 10.3$ & 0.317 & $56.2 \pm 12.6$ & $55 \cdot 3 \pm 12.1$ & 0.673 \\
\hline \multicolumn{7}{|l|}{ Sex } \\
\hline Male & 50 & 43 & - & 44 & 41 & - \\
\hline Female & 24 & 32 & - & 23 & 23 & - \\
\hline Body surface index & $23.97 \pm 3.50$ & $23.54 \pm 2.84$ & 0.411 & $23.54 \pm 3.21$ & $24.02 \pm 3.85$ & 0.441 \\
\hline \multicolumn{7}{|c|}{ ASA physical status classification } \\
\hline 1 & 50 & 57 & - & 45 & 47 & - \\
\hline 2 & 19 & 18 & - & 22 & 15 & - \\
\hline 3 & 5 & 6 & - & o & 2 & - \\
\hline
\end{tabular}

Values are presented as mean $\pm \mathrm{SD}$ or number.

BIS, bispectral index; MOAA/S, modified Observer's Assessment of Alertness/Sedation Scale; ASA, American Society of Anesthesiologists.

\section{Propofol titration dose and BIS value}

The mean BIS value throughout the procedure (from insertion to removal of the endoscope) was $74.3 \pm 6.7$ for all 141 patients in the BIS group. The mean total propofol dose administered in the BIS group was significantly higher than that in the MOAA/S group $(36.9 \pm 29.6$ and $11.3 \pm 20.7$, respectively; $p<0.001$ ), in both the expert and inexperienced endoscopist groups (expert group: $38.8 \pm$ 25.6 and $10.5 \pm 15.8 \mathrm{mg}$, respectively, $p<0.001$; inexperienced group: $34.8 \pm 33.5$ and $12.2 \pm 25.3 \mathrm{mg}$, respectively, $p<$ o.001). The difference between the two groups remained significant after adjusting for patient body surface index and procedure duration (Table 2). However, the total dose of propofol administered in the inexperienced endoscopist group did not differ significantly from that in the expert endoscopist group, with or without the use of $\mathrm{BIS}(p=0.430$ and $p=0.640$, respectively). The procedure time was longer in the inexperienced endoscopist group than in the expert group, with or without the use of BIS $(27.8 \pm 14.5$ minutes vs. $20.1 \pm 12.4$ minutes, $p=0.001 ; 28.4$ \pm 14.3 minutes vs. $17.5 \pm 7.6$ minutes, $\mathrm{p}<0.001$ ).

\section{Patient satisfaction and adverse events}

The degree of pain assessed by VAS score during colonoscopy did not differ significantly between the BIS and MOAA/S groups in either the expert or inexperienced group $(1.38 \pm 1.03$ vs. $1.56 \pm 0.95, p=0.264 ; 1.93 \pm 2.10$ vs. $2.14 \pm 1.75, p=0.526$, respectively). Although overall patient satisfaction during colonoscopy was higher in the expert group than in the inexperienced group, with or without the use of BIS $(p<0.001$ and $p<0.001)$, the differences between the BIS and MOAA/S groups in the expert and inexperienced groups were likewise not significant (Table 2). No adverse events, including frequency of oxygen saturation $<80 \%$, hypotension $(<80$ $\mathrm{mmHg}$ ), or bradycardia ( $<50$ beats per minute), were observed. The endoscopists' satisfaction was excellent for all patients in each group. During the post-procedural follow-up assessment performed in the recovery area, no clinically significant hypoxic episodes were recorded in either group.

\section{DISCUSSION}

In the current study, we found that during colonoscopy examination the BIS group did not differ significantly from the MOAA/S group in terms of endoscopist or patient satisfaction. However, the propofol dose administered was higher in the BIS group than in the MOAA/S group. These results were not significantly different between the expert and inexperienced endoscopist groups.

Previous studies have reported the usefulness of BIS monitoring in endoscopic submucosal dissection or ERCP, as well as significant advantages in terms of the propofol dose administered, and in endoscopist and patient satisfaction levels $[4,14,15]$. However, in previous studies that targeted deep sedation during screen- 
Table 2. Sedative dose, procedure time, and satisfaction scores

\begin{tabular}{|c|c|c|c|c|c|c|}
\hline \multirow{2}{*}{ Characteristic } & \multicolumn{3}{|c|}{ Expert endoscopist } & \multicolumn{3}{|c|}{ Inexpert endoscopist } \\
\hline & $\operatorname{BIS}(\mathrm{n}=74)$ & MOAA/S (n = 75) & $p$ value & $\operatorname{BIS}(n=67)$ & $\mathrm{MOAA} / \mathrm{S}(\mathrm{n}=64)$ & $p$ value \\
\hline Midazolam, mg & $3.16 \pm 0.62$ & $3.17 \pm 0.62$ & 0.913 & $3.10 \pm 0.43$ & $3.03 \pm 0.36$ & 0.292 \\
\hline \multicolumn{7}{|l|}{ Propofol, mg } \\
\hline Total dose & $38.8 \pm 25.6^{\mathrm{a}}$ & $10.5 \pm 15.8^{b}$ & $<0.001$ & $34.8 \pm 33.5^{\mathrm{a}}$ & $12.2 \pm 25.3^{b}$ & $<0.001$ \\
\hline Total dose/BSA & $1.664 \pm 1.148$ & $0.507 \pm 0.716$ & $<0.001$ & $1.468 \pm 1.398$ & $0.503 \pm 1.026$ & $<0.001$ \\
\hline Total dose/BSA/total time & $0.097 \pm 0.070$ & $0.026 \pm 0.037$ & $<0.001$ & $0.057 \pm 0.055$ & $0.016 \pm 0.031$ & $<0.001$ \\
\hline \multicolumn{7}{|l|}{ Procedure time, min } \\
\hline Cecal intubation & $7.0 \pm 4.6$ & $7.1 \pm 4.8$ & 0.849 & $12.5 \pm 7.3$ & $12.4 \pm 7.9$ & 0.976 \\
\hline Total & $20.1 \pm 12.4^{c}$ & $17.5 \pm 7.6^{\mathrm{d}}$ & 0.127 & $27.8 \pm 14.5^{c}$ & $28.4 \pm 14.3^{\mathrm{d}}$ & 0.830 \\
\hline \multicolumn{7}{|l|}{ Patients' VAS scores ${ }^{\mathrm{e}}$} \\
\hline Pain during colonoscopy & $1.38 \pm 1.03$ & $1.56 \pm 0.95$ & 0.264 & $1.93 \pm 2.10$ & $2.14 \pm 1.75$ & 0.526 \\
\hline Overall satisfaction & $9.35 \pm 1.18^{f}$ & $9.31 \pm 1.07^{\mathrm{g}}$ & 0.808 & $6.25 \pm 3.97^{f}$ & $5.61 \pm 3.75^{\mathrm{g}}$ & 0.342 \\
\hline
\end{tabular}

Values are presented as mean $\pm \mathrm{SD}$.

BIS, bispectral index; MOAA/S, modified Observer's Assessment of Alertness/Sedation Scale; BSA, body surface area; VAS, visual analog scale.

${ }^{\mathrm{a}, \mathrm{b}}$ The dose of propofol administered was not significantly different between the inexperienced and expert endoscopist groups, both with and without the use of BIS ( $p=0.430$ and $p=0.640$, respectively).

${ }^{c, d}$ The procedure time was longer in the inexperienced endoscopist group than in the expert group, both with and without the use of BIS ( $p=0.001$ and $p<0.001$, respectively).

${ }^{\mathrm{e}} \mathrm{A}$ VAS was used to score pain (o, none; 10, severe) and satisfaction (o, poor; 10, excellent).

f,g The patient's overall satisfaction during colonoscopy was higher in the expert group than in the inexperienced group, both with and without the use of BIS $(p<0.001$ and $p<0.001$, respectively).

ing endoscopy, no significant differences in propofol dose or recovery time were found between the BIS and MOAA/S groups $[5,6]$. Similarly, in the present study, which involved moderate sedative colonoscopy, patient satisfaction and propofol titration dose did not differ markedly between the BIS and MOAA/S groups. Further, the results indicate that BIS may be more appropriate in the maintenance phase of sedation, rather than in the induction or recovery phase [5]. Likewise, an important point for application of BIS in the sedative endoscopic field may be the duration, not the depth, of sedation. Therefore, BIS could be more useful when a longer duration of sedation is required, such as during therapeutic endoscopic procedures.

BIS values range from $\mathrm{o}$ (coma) to 100 (fully awake), and reflect the sedation level regardless of patient clinical characteristics or the type of sedative drug administered [16]. In previous studies, BIS was validated for monitoring sedation, as evidenced by the good correlation with sedation level $[10,17]$. In this study, we set the threshold BIS score at 70 to 80 for moderate sedation based on prior recommendations for using BIS during colonoscopy [9]. However, in other studies, a BIS score of 80 was the approximate standard for moderate sedation $[10,17]$. Therefore, in our study, the higher propofol dose in the BIS group may have been related to the lower BIS score setting for moderate sedation. Nonetheless, the spectrum of changes from moderate to deep sedation may be very narrow. In a previous study, the BIS score for moderate sedation significantly overlapped with that for deep sedation [18]. Although further research is needed to determine the BIS score for moderate sedation, maintaining the BIS set point during sedative colonoscopy may be too difficult in clinical practice.

Because the need for endoscopic examinations is increasing, the use of sedatives, propofol in particular, has also increased [19]. However, in some countries, the use of a sedative agent is restricted and can only be administered by an anesthesiologist during colonoscopy. In this situation, the concern is the high cost. In a recent study from France, sedative colonoscopy, performed under sedation by an anesthesiologist, added $285 \%$ to 
the cost of nonsedative colonoscopy [20]. On the other hand, nonanesthesiologist administration of propofol showed greater safety and satisfaction levels compared with anesthesiologist-administered colonoscopy [21]. Interestingly, in our study, the propofol dose administered did not differ significantly between the expert and inexperienced endoscopist groups, irrespective of use of BIS ( $38.8 \pm 25.6$ vs. $34.8 \pm 33.5, p=0.430)$, or MOAA/S (10.5 \pm 15.9 vs. $12.1 \pm 25.3, p=0.640)$. Moreover, there was no adverse event during colonoscopic examination in either group. The results suggest that the administration of combined sedation by a well-trained nurse could be considered a safe and satisfactory method for sedative colonoscopy.

The limitations of the current study included the fact that the nurses could not be blinded to the absence or presence of BIS monitoring during colonoscopy. However, it was impossible for this study to be performed in a totally blinded fashion. While additional, well-designed, double-blinded studies should be conducted to confirm our results, the implication is that sedation administered by well-trained nurses is efficacious in sedative colonoscopy. A second limitation of the study was that we did not investigate or set the BIS score for moderate sedation in accordance with the results of previous studies. Although further research is needed, we believed that setting a different BIS score for moderate sedation would not reduce the propofol dose titration. Finally, although we observed no adverse events during the procedures, we could not definitively conclude whether or not the experience level of the medical team conducting the sedation procedures had an effect on the complication rate.

In conclusion, compared with MOAA/S score monitoring alone, BIS monitoring was not effective for titrating the dose of propofol in colonoscopy, irrespective of endoscopist experience.

\section{KEY MESSAGE}

1. The bispectral index monitoring method doesn't seem like better than modified Observer's Assessment of Alertness/Sedation Scale (MOAA/S) when the trained nurse monitored in moderate sedation.

2. The moderate sedative colonoscopy could be well performed by the trained nurse using MOAA/S even though the operator is less experienced.

\section{Conflict of interest}

No potential conflict of interest relevant to this article was reported.

\section{Acknowledgments}

We thank all of the investigators and study personnel for their contributions. This work was supported by a grant from the Biomedical Research Institute, Kyungpook National University Hospital (2012).

\section{REFERENCES}

1. Jung HS, Park DK, Kim MJ, et al. A comparison of patient acceptance and preferences between CT colonography and conventional colonoscopy in colorectal cancer screening. Korean J Intern Med 2009;24:43-47.

2. Schutz SM, Lee JG, Schmitt CM, Almon M, Baillie J. Clues to patient dissatisfaction with conscious sedation for colonoscopy. Am J Gastroenterol 1994;89:1476-1479.

3. Soehle M, Ellerkmann RK, Grube M, et al. Comparison between bispectral index and patient state index as measures of the electroencephalographic effects of sevoflurane. Anesthesiology 2008;109:799-805.

4. Jang SY, Park HG, Jung MK, et al. Bispectral index monitoring as an adjunct to nurse-administered combined sedation during endoscopic retrograde cholangiopancreatography. World J Gastroenterol 2012;18:6284-6289.

5. Chen SC, Rex DK. An initial investigation of bispectral monitoring as an adjunct to nurse-administered propofol sedation for colonoscopy. Am J Gastroenterol 2004;99:1081-1086.

6. Drake LM, Chen SC, Rex DK. Efficacy of bispectral monitoring as an adjunct to nurse-administered propofol sedation for colonoscopy: a randomized controlled trial. Am J Gastroenterol 2006;101:2003-2007.

7. Leslie K, Absalom A, Kenny GN. Closed loop control of sedation for colonoscopy using the bispectral index. Anaesthesia 2002;57:693-697.

8. Ni YF, Li J, Wang BF, et al. Effects of electroacupunc- 
ture on bispectral index and plasma beta-endorphin in patients undergoing colonoscopy. Zhen Ci Yan Jiu 2009;34:339-343.

9. von Delius S, Thies P, Rieder T, et al. Auditory evoked potentials compared with bispectral index for monitoring of midazolam and propofol sedation during colonoscopy. Am J Gastroenterol 2009;104:318-325.

10. Yu YH, Han DS, Kim HS, et al. Efficacy of bispectral index monitoring during balanced propofol sedation for colonoscopy: a prospective, randomized controlled trial. Dig Dis Sci 2013;58:3576-3583.

11. Keats AS. The ASA classification of physical status: a recapitulation. Anesthesiology 1978;49:233-236.

12. Cass OW, Freeman ML, Peine CJ, Zera RT, Onstad GR. Objective evaluation of endoscopy skills during training. Ann Intern Med 1993;118:40-44.

13. Rex DK. Review article: moderate sedation for endoscopy: sedation regimens for non-anaesthesiologists. Aliment Pharmacol Ther 2006;24:163-171.

14. Paspatis GA, Chainaki I, Manolaraki MM, et al. Efficacy of bispectral index monitoring as an adjunct to propofol deep sedation for ERCP: a randomized controlled trial. Endoscopy 2009;41:1046-1051.

15. Imagawa A, Fujiki S, Kawahara Y, et al. Satisfaction with bispectral index monitoring of propofol-mediated sedation during endoscopic submucosal dissection: a prospective, randomized study. Endoscopy 2008;40:905-909.

16. Johansen JW. Update on bispectral index monitoring. Best Pract Res Clin Anaesthesiol 2006;20:81-99.

17. Bower AL, Ripepi A, Dilger J, Boparai N, Brody FJ, Ponsky JL. Bispectral index monitoring of sedation during endoscopy. Gastrointest Endosc 2000;52:192-196.

18. Qadeer MA, Vargo JJ, Patel S, et al. Bispectral index monitoring of conscious sedation with the combination of meperidine and midazolam during endoscopy. Clin Gastroenterol Hepatol 2008;6:102-108.

19. Heuss LT, Froehlich F, Beglinger C. Changing patterns of sedation and monitoring practice during endoscopy: results of a nationwide survey in Switzerland. Endoscopy 2005;37:161-166.

20. Hassan C, Benamouzig R, Spada C, et al. Cost effectiveness and projected national impact of colorectal cancer screening in France. Endoscopy 2011;43:780-793.

21. Poincloux L, Laquiere A, Bazin JE, et al. A randomized controlled trial of endoscopist vs. anaesthetist-administered sedation for colonoscopy. Dig Liver Dis 2011;43:553558. 